\title{
Quelques observations sur la parenté spirituelle en Normandie (Xe-XIle siècle)
}

In: Cahier des Annales de Normandie n³2, 2002. Mélanges Pierre Bouet. pp. 81-91.

Citer ce document / Cite this document :

Bauduin Pierre. Quelques observations sur la parenté spirituelle en Normandie (Xe-Xlle siècle). In: Cahier des Annales de Normandie ${ }^{\circ} 32$, 2002. Mélanges Pierre Bouet. pp. 81-91.

http://www.persee.fr/web/revues/home/prescript/article/annor_0570-1600_2002_hos_32_1_2435 


\section{QUELQUES OBSERVATIONS SUR LA PARENTÉ SPIRITUELLE EN NORMIANDIE $\left(X^{\mathrm{c}}-\mathrm{XII} I^{\mathrm{C}}\right.$ SIÈCLE)}

l.ors du concile de lillebonne (los(1). I laglise normande rapplat les interdits pesant sur les relations charnelles entre des indis idus lies par une parente spirituelle. celles-ci elaient proserites entre un homme et sal marraine sa commere ou sa

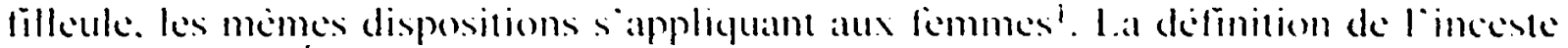
spirituel par loglise a depuis longlemps rekenu lattention des juristes et des historiens du droit. gui se sont attaches a en souligner leincidence sur la legistation canonicue du mariages. Des recherches conduites dans une perspective plus anthropologigue se sont attacheses a en digager les fondements idech et ont permis de faire ressortir l’importance et looriginalite des liens spirituels dams ke systeme de parenti midisial:

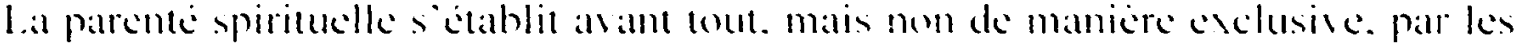
liens (parrainage el comperage qui se mouent a loceasion de la ciremonie du

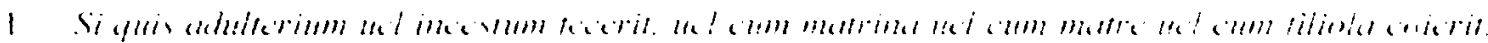

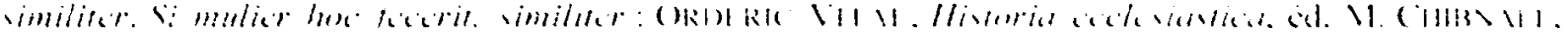

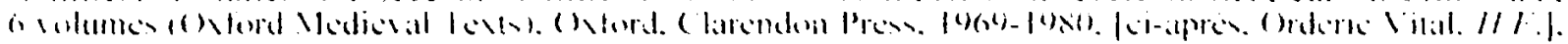
$\checkmark$.

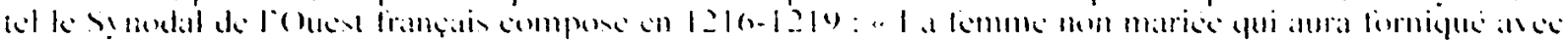

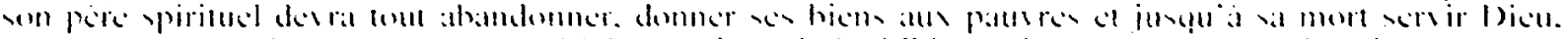

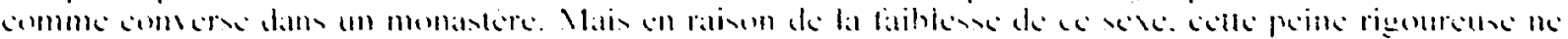

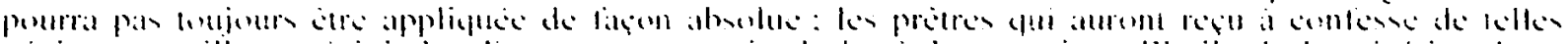

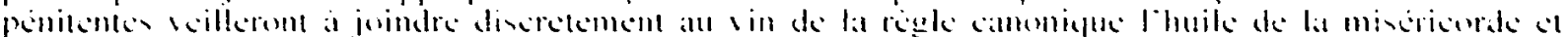

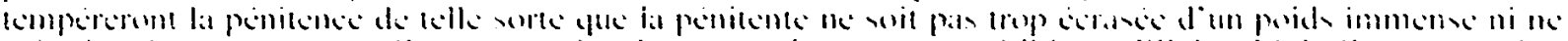

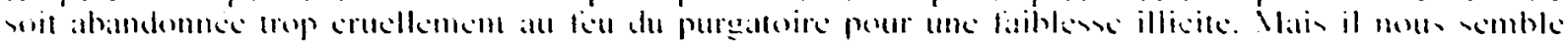

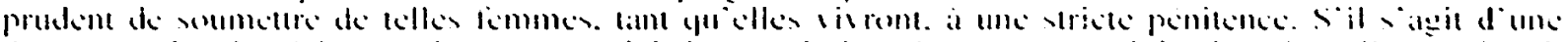

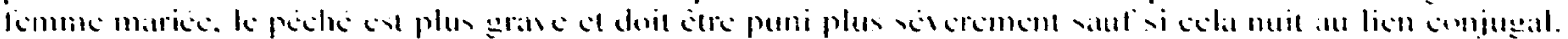

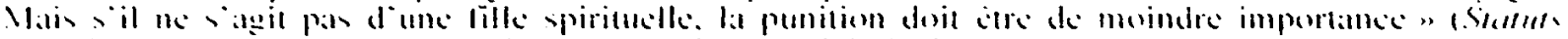

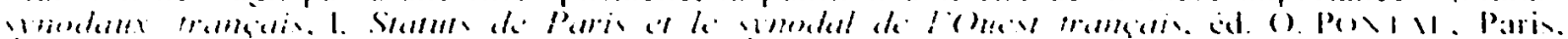

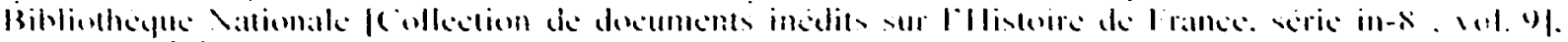
1171. P. $202-2(1+1$.

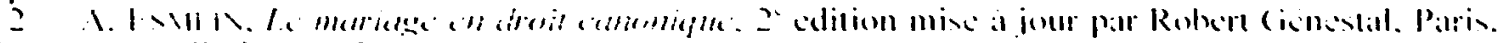

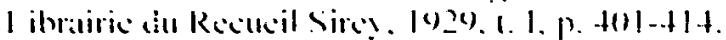

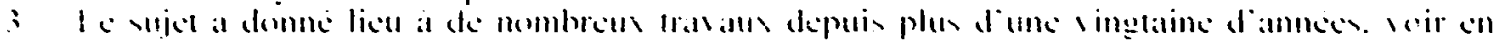

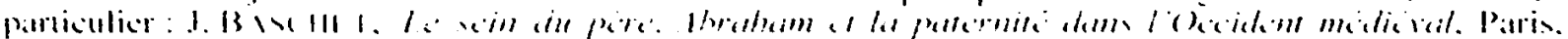

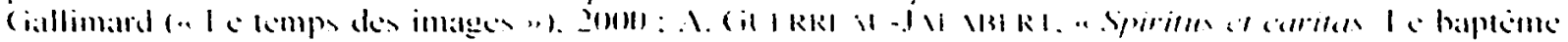

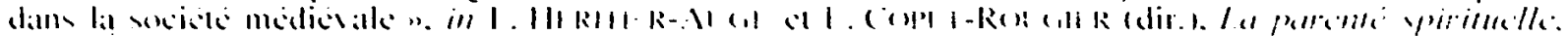

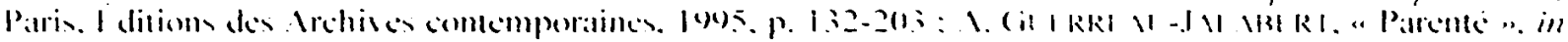

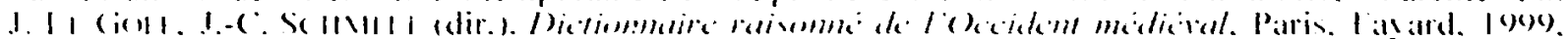

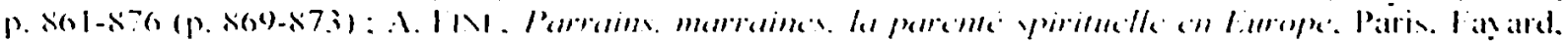

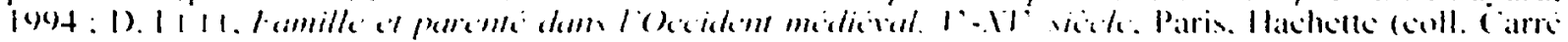

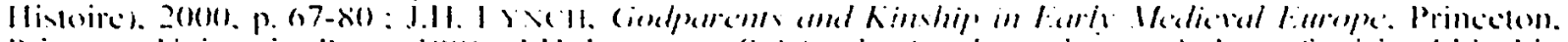

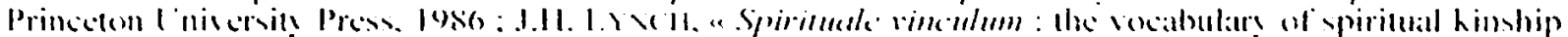

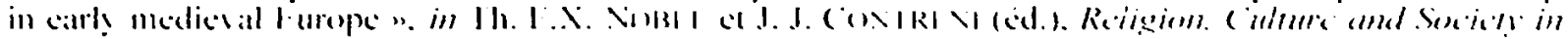

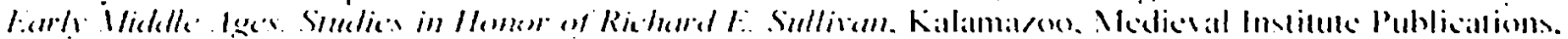
1487. p. $181-201$. 
haptèmet. Dégagéc du péché de chair. elle est considéré par l’liglise comme supéricure à la parenté biologique et promue comme un modele de relations sociales. llle a aussi produit des pratiques que s'est approprié la societé, parfois au prix d'une distorsion de l'idéal ecelésiastique. L’analyse de ces pratiques sociales est un domaine d'étude désormais bien balisé par des travaux qui concernent le début et la fin de la période médiévale.'. Pour la Normandie des $X^{\circ}-X 11^{\circ}$ siecles, elle reste un sujet à peu près vierge.

Les relations créées par le baptême ont engendré un vocabulaire spécifique. construit a partir de termes qui font référence a la filiation biologique filiolus filiola: lillcul(c), patrimus / matrina: parrain / marraine. compater : commater: compère / commère). (ette terminologie mit du temps à s`imposer. (On sait par exemple que patrints et matrina ne sont pratiquement jamais employés avant le VIII" siècle, les auteurs médiévaux préfërant utiliser des expressions telles que parer spiritualis ou pater ex lancaro, ces hésitations lexicales semblant toutefois avoir eté moins sensibles pour désigner les relations de compérage". Les sources normandes des $X^{\prime \prime}-X 11^{\circ}$ siècles usent volontiers de périphrases qui décrivent l'action du (ou des) parrain(s) au moment de la cérémonic baptismale, en particulier lorsque celui-ci "live" ou "reçoit " le nouveau chrétien au sortir des fonts?. Palrimus désigne

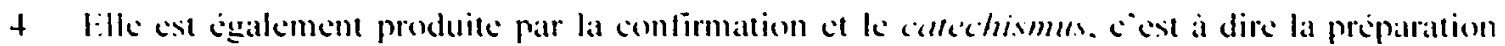

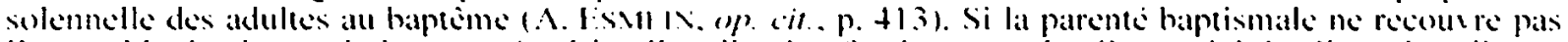
lensemble du champ de la parente spirituelle. elle ne fonde pas moins lessentiel des liens dont il sera yucstion ici.

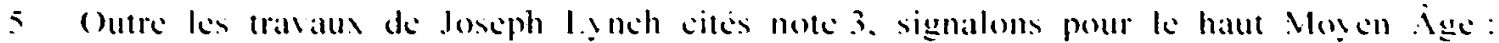

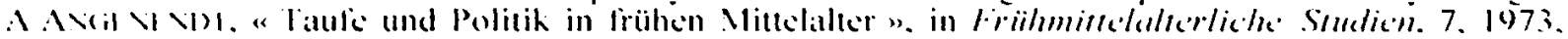

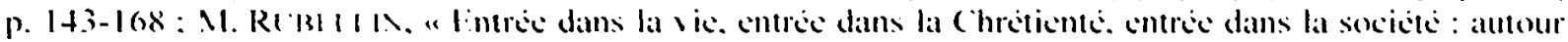

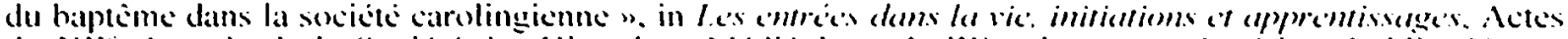

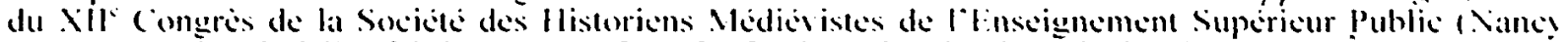

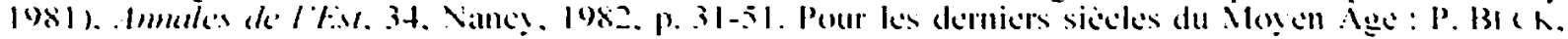

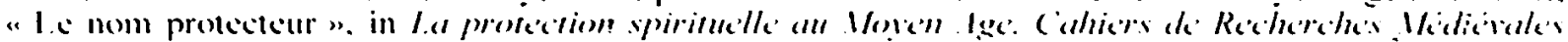

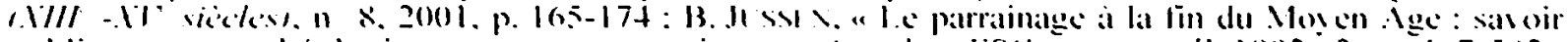

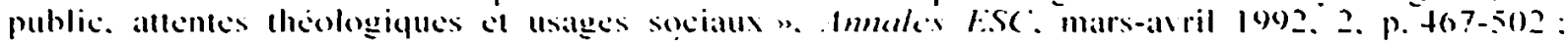

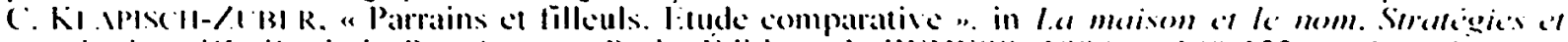

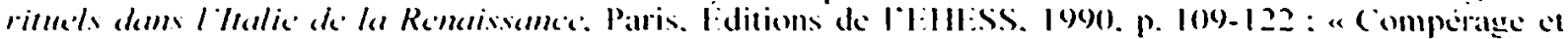
clientilisme". Ihid. p. 123-133: (h. K1 APlsch-/.1 131 R. "Au peril des commeres. I alliance spirituelle

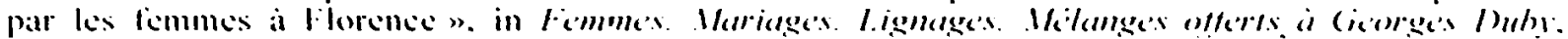

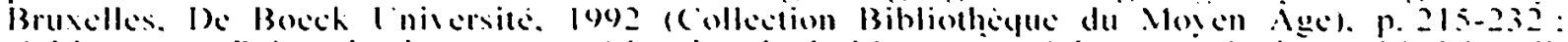

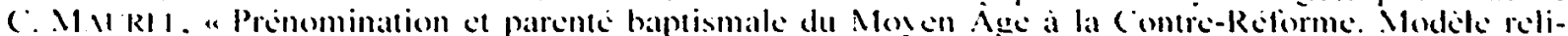

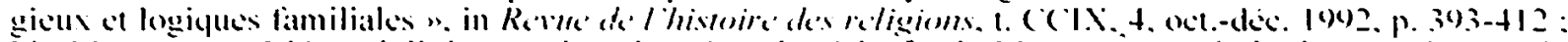

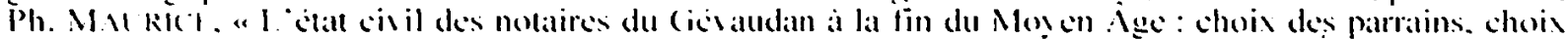

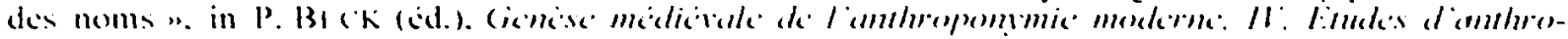

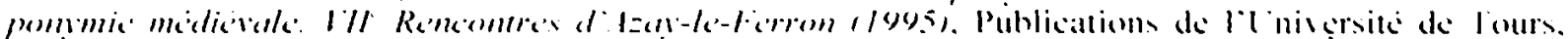

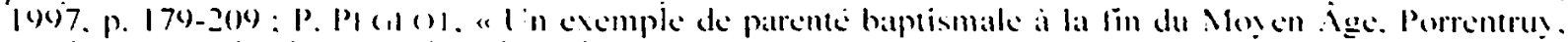

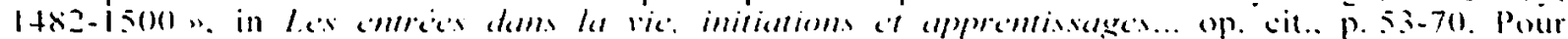

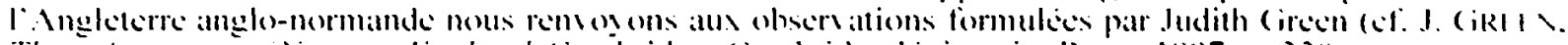

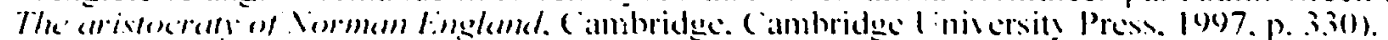

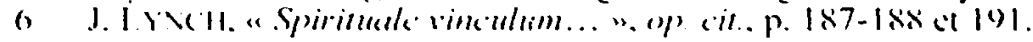

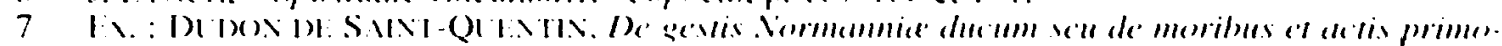

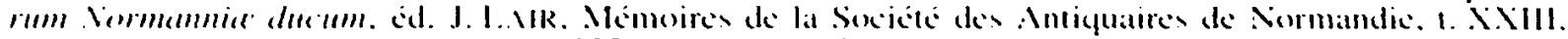

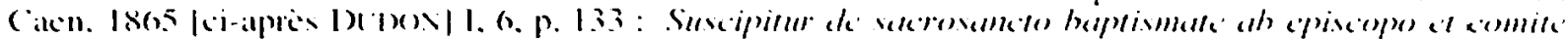

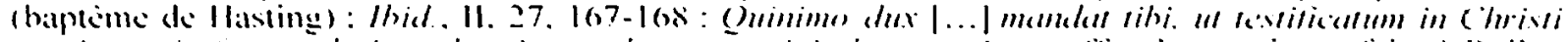

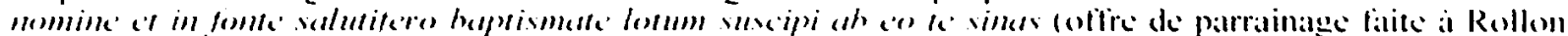

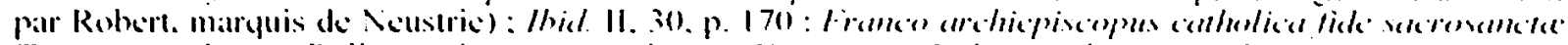

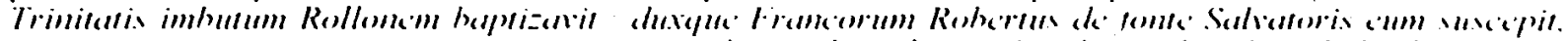

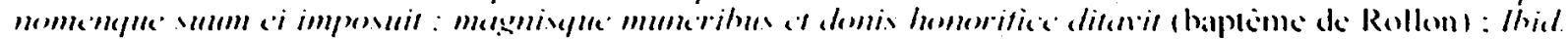

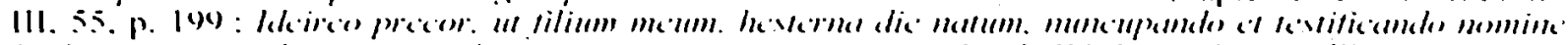

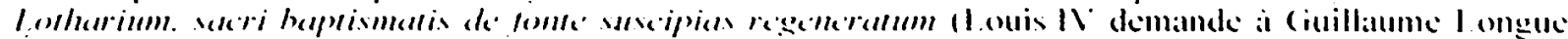

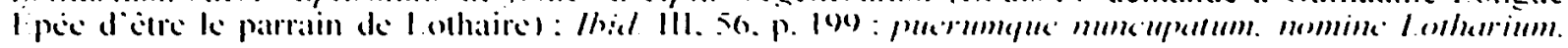


partois le prêtre qui a administré le sacrement. du moins si l'on se réfëre à l'un des passages autobiographicues de l'Histoire Ecelesicostique, où (Orderic Vital rappelle qu’il a "été baptisé le samedi de Páques à Atcham, bourg situé en Angleterre sur les bords du grand tleure qu ist la Severn |... La. par le ministere du prêtre (Orderic, tu mas régenére de l'eau de l’lesprit Saint et tu mas donne le nom de ce prêtre, mon parrain $" *$. Il est vai qu un prêtre peut être le parrain d"un enfant qu"il baptise". Dans un mème orde d idés, filiolus peut qualifier le baptise par rapport a l homme

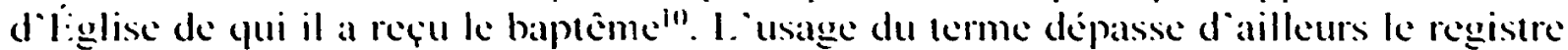
des liens crés par le baptême : éest en les appelant filioli yue saint Taurin, dans son tombeau. sadresse aux habitants de la cite d'ireux" ou que labbe firoul transmet ses ultimes recommandations à ses moines peu avant de mourir'2. ()n sait que la liliation spirituelle pouvait être insoquée en d'autres circonstances, comme en

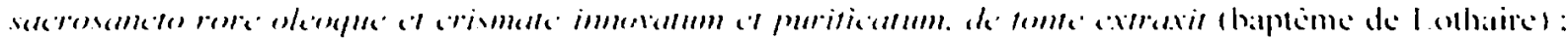

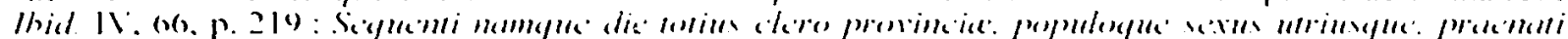

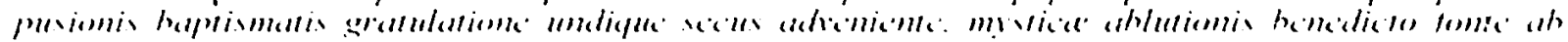

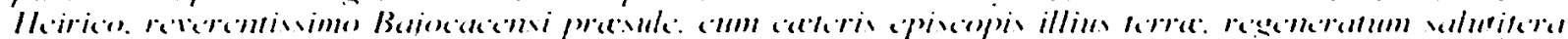

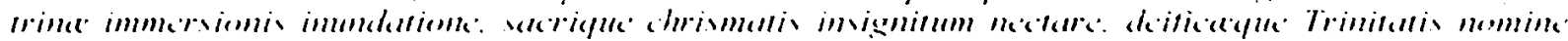

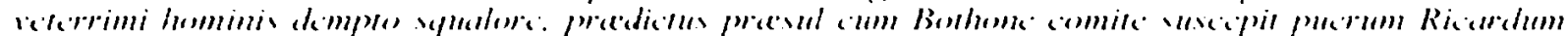

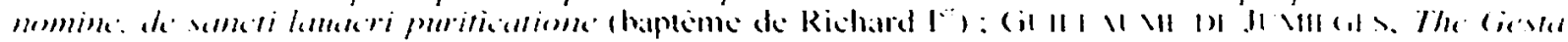

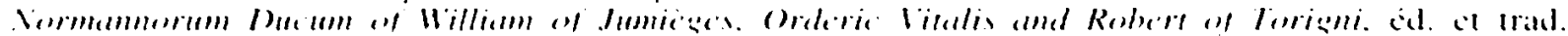

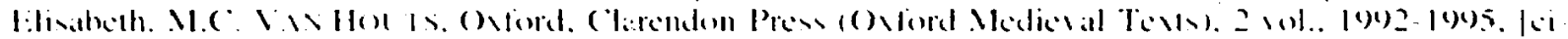

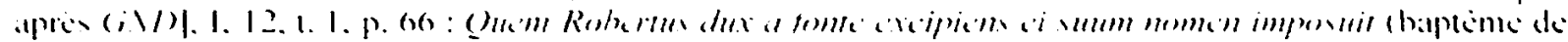

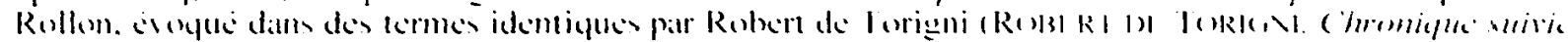

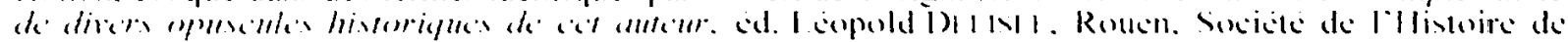

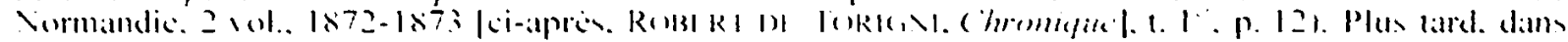

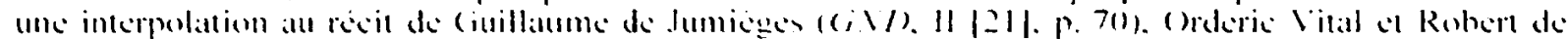

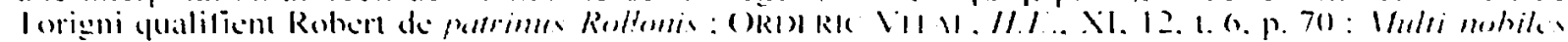

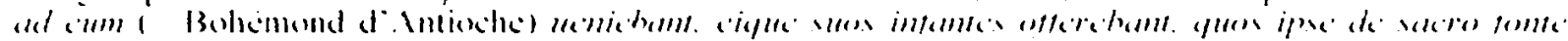

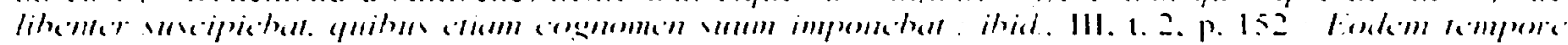

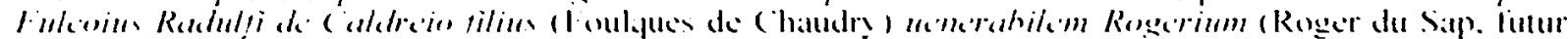

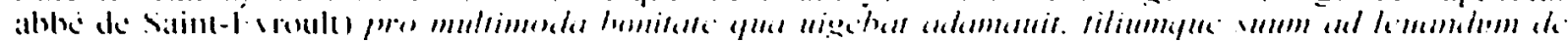

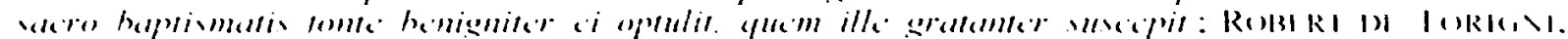

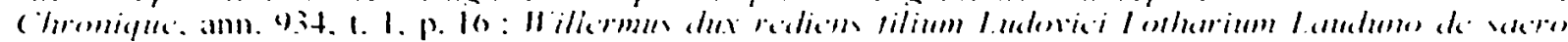

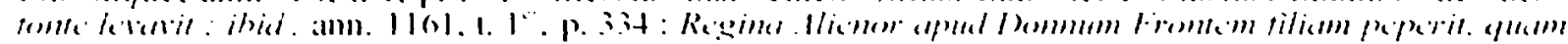

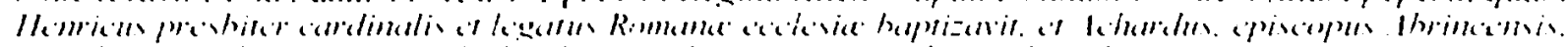

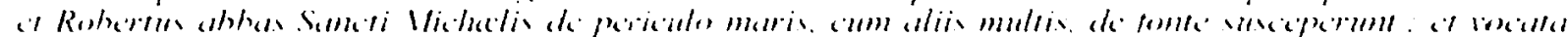

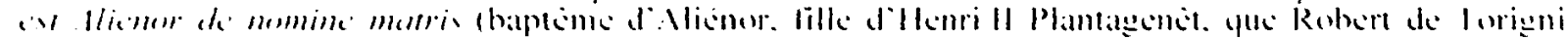

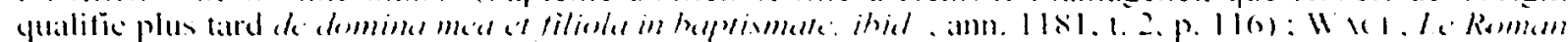

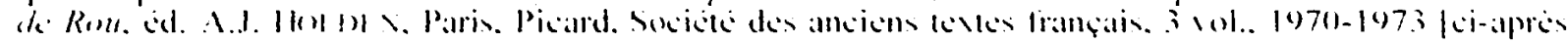

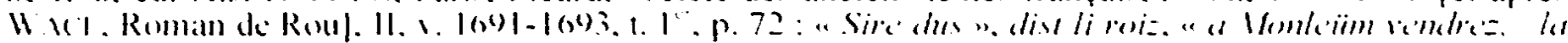

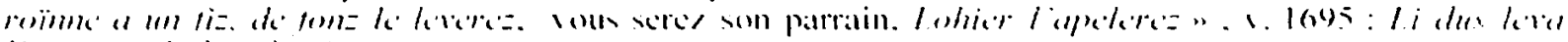
linfunt a lohicr li mivi nom.

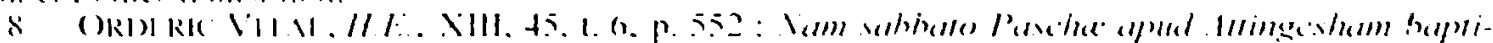

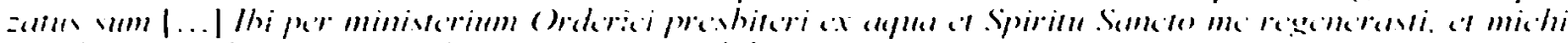

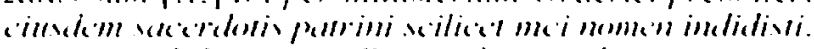

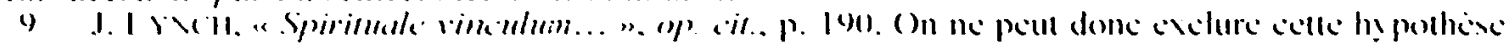
dams ke can du pritre (Moleric.

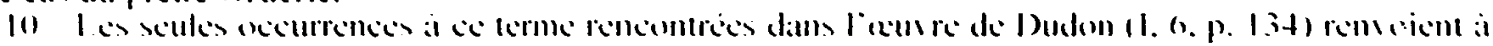

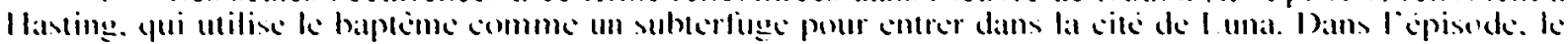

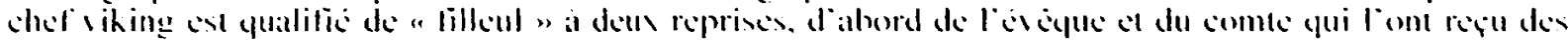

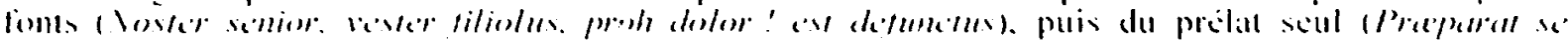

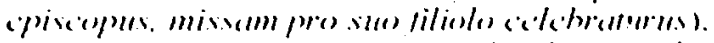

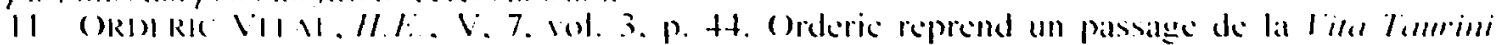

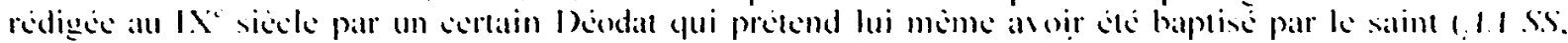

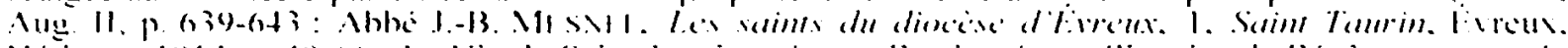

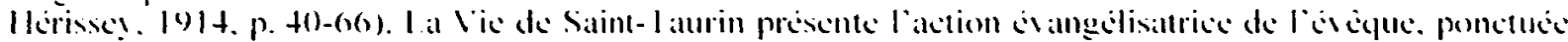

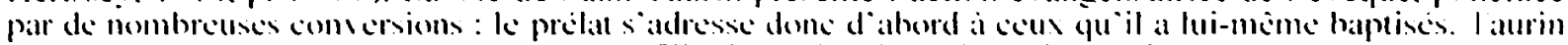

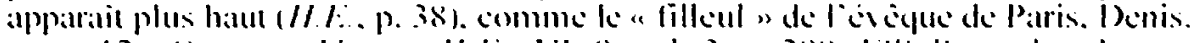

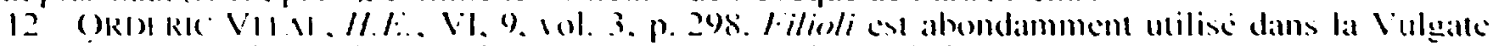

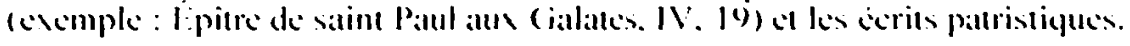


lémoigne par exemple la correspondance de Lantrance "consciller spirituel " de la reine Marguerite d licossel:

Nos sources se montrent fort discretes sur les pratiques liés au parrainage. Quel était le nombre de parrains/marraines? La documentation consultée ne live le plus sourent qu un seul nom. toujours un homme. Toutefois Alienor, fille d'llenri II Plantagenêt, en eut plusieurs : Achard. érêue d'Avranches et Robert de Torigni. abbé du Mont-Saint-Michel, cum aliis multis: ${ }^{1+}$; 4 un enfant pouvait etre parrains par une communauté religieuse tout entierels. I)ans la mesure où il est possible de déterminer les origines familiales des intéressés, il semble que le choix du parrain ait été opéré en dehors de la parenté charnelle. Le baptême s’acompagnait de dons remis par le parrain à lissue de la cérémonic. Dudon de Saint (Quentin souligne ainsi la magnificence des présents octroyés par le marquis Robert a son filleul Rollon ou par (iuillaume Longue lipe au jeune Lothaire ${ }^{16}$. De maniere plus indirecte, la relation créce par le haptême pouvait être perçue comme un facteur fatvorisant ce type déchanges. Orderic Vital relic ainsi la donation de l"église de Parnes a laffection (dilectio) croissante issue du compérage établi entre foulques de Chaudry et Roger du Sap. futur abbe de Saint-livroult?"

La fonction des parents spirituels apparait simplement en filigrane. Il est difficile d'établir dans quelle mesure le parrain influençait les choix présidant à l'avenir de son tilleul. (Orderic Vital rapporte que le pape pascal Il avait ordonné que son filiolus. Pons de Melgueil. fût élevé à Cluny"s. De mème. la rocation monastique de Thierry, futur abbé de Saint-livroult, semble devoir beaucoup à son parrain Thierry. abbe de Jumieges, et lon peut se demander si Roger du Sap n’a pas orienté la carriere de son filleul" ${ }^{10}$. Selon Dudon. (iuillaume Longue fipée envoya le jeune Richard $l^{\text {cr }}$ a Baycux pour cu il apprenne la langue norroise et contia son éducation

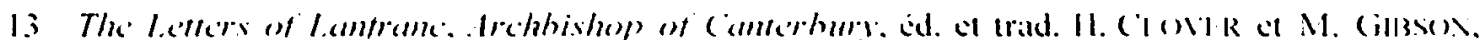

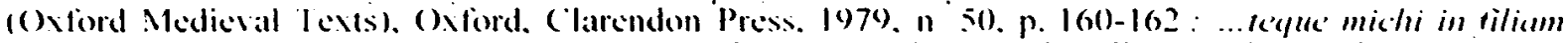

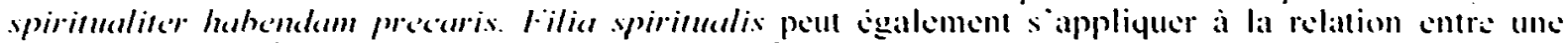
femme et son contesseur (A. Esill I. op. cit. . p. +13 ).

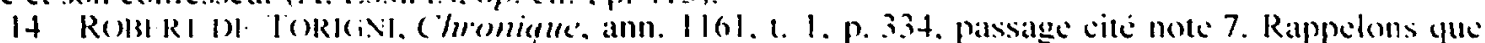
lighlise a rapidement limits le nombe de parents spirituels. en pronant un chiffec impair, deabord a un seuil parrain (le concile de Met/ de 888 ne dit rien sur les marraines). puis a trois parents spiritucls.

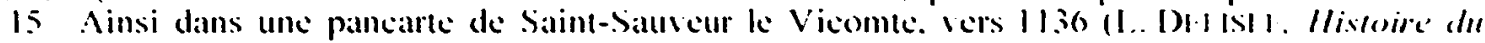

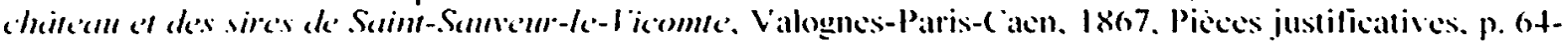

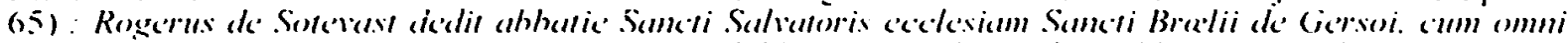

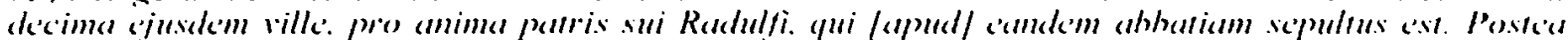

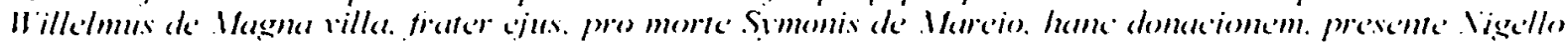

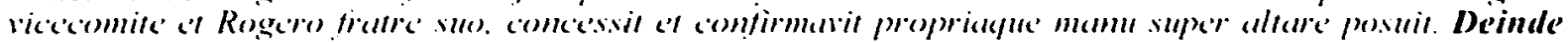
Johannes, filius ejus, et filiolus ommium monachorum. hanc donacionem in vitu patris sui et post

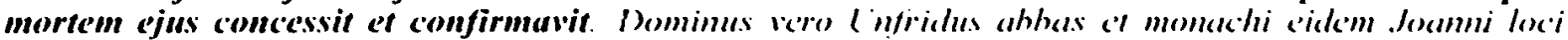

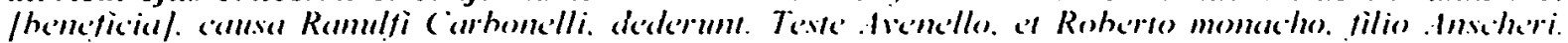

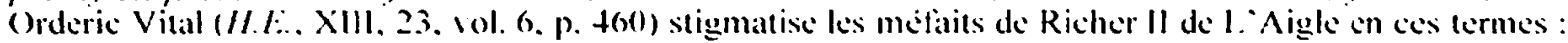

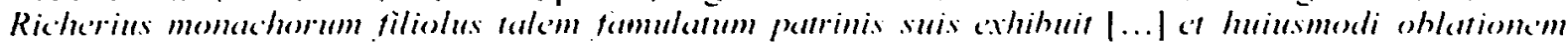

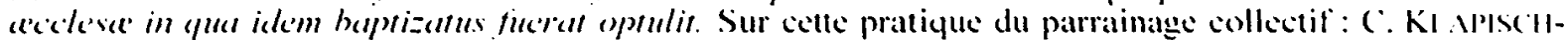
7.1 B1-R. "Parrains et filleuls..." " op. cit. p. $116-117$ et note $33:$ " Comperage et clientélisme..." op. (it..p. 127.

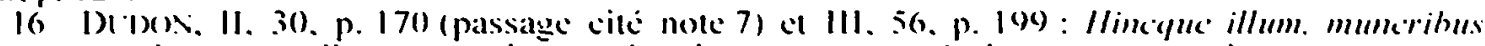

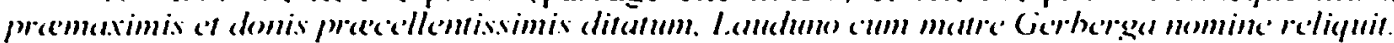

17 ()RI) RIC VIIAI . II. Livre III. 1. 2. p. 152. Parnes, canton de (haumont-en-Vexin. ()ise.

Is thid., Xll, 21. 1. 6. p. 268.

19 Pour Thierry : Thid., III, t. 2. p. I8. Orderic Vital n indicpue pas ke nom du filleul de Roger. mais il faut peut atre lidentitier comme lun des deux lils de lonlques de (hatudry qui furent moines a Saint-livroult tihidl. III. 1. 2. p. I54). 
atl parrain de lenfant, Bothon"0". Pour interessantes qu elles soient, ees indications ne sultisent pas pour demontrer que les parents spirituds avaient pour vocation de se substituer aux parents biologicues.

De la meme maniere, aucun dispositil legal ne presoyat la transmission des biens en faseur du lilleul. (On notera toulefois yue celle presocupation nest pas totalement absente. (lne notice du cartulaire de la Triniti-du-Mont rapporte ainsi la donation d'une terre faite dans le troisieme yuart du $\mathrm{Xl}^{\circ}$ siecle par un certain lides et son épouse limengarde, reserrant la jouissance du bien, pour une moitie au conjoint survivant el pour l'autre moitic anec une maison dans le laubourg /de Rouen|, aux entants du couple el a leurs stlcessseurs. Toutefois il est consenu qui en l'absence d'heritier vivant. la maison reviendrait a la tille qu limengarde arat eue

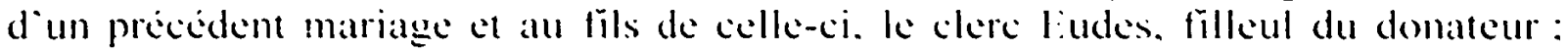
apres yuoi la maison retoumerait au monastere de la Trinite-du-Montel. Le cas est interessant a double titre. 1) une part. car il nous informe sur le choix du parrain.

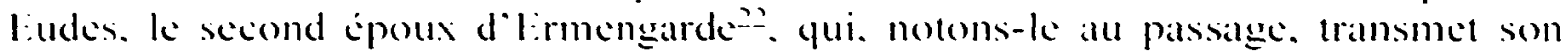
nom a son tilleul. In second lieu. parce yue cest dabord la relation spirituelle entre les deux personnages qui est insoquéc pour accorder la jouissance du bien². Les raisons de ees considerations nous echappent. Des dispositions semblables ne sont pas inconnues:- de méme yue les praticyues consistant. pour le parrain, à donner des

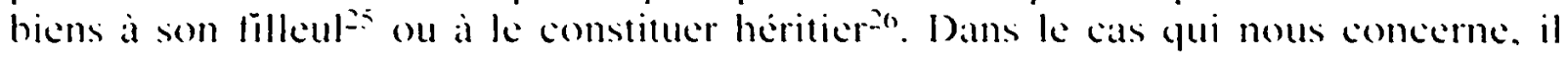
serait toutefois temtant dy voir un dispositif permeltant de reintroduire le clere liudes el sa mere dans un cercle d’ayants droit dont ils etaient a priori exclus.

Nous sommes à peine mieux renseignes sur le rôle des parents spirituels dans la transmission du nom. Les donnés disponibles restent trop fragmentaires pour permettre une quelconyuc généralisation, et nous nous lis rerons d autant moins à lexercice qüil manque encore une synthese permettant devaluer, pour la Norman-

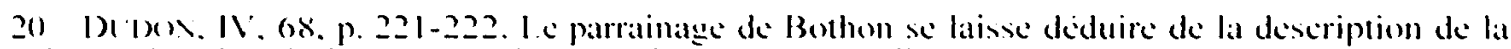

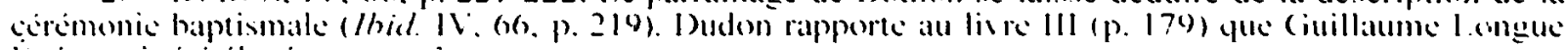

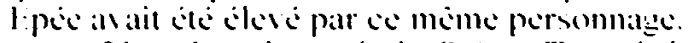

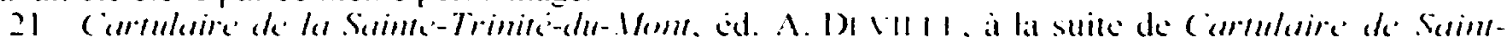

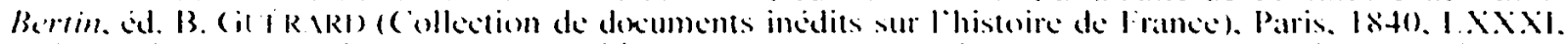

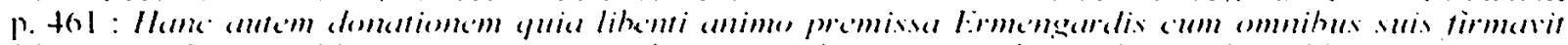

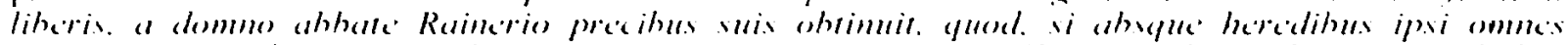

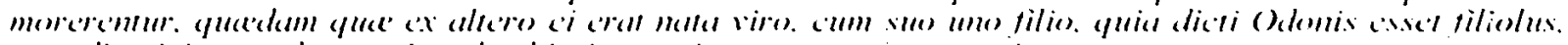

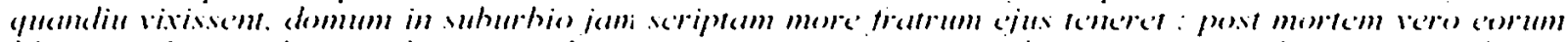

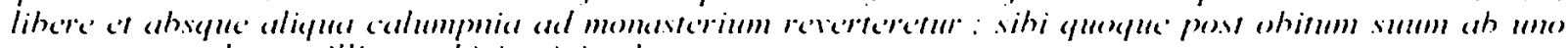

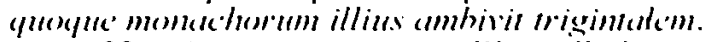

22 (On remarquera que linterdit frappant lunion entre un parrain et sa commere ne sapplique pas ici : én est pas limengarde mais sa fille cui est la commire diludes.

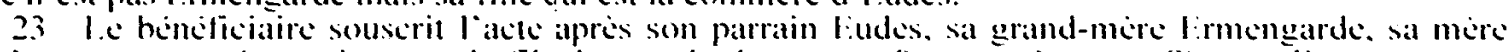

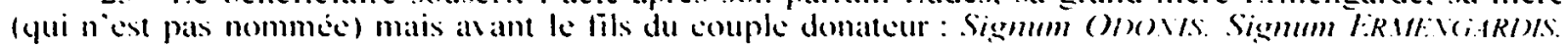

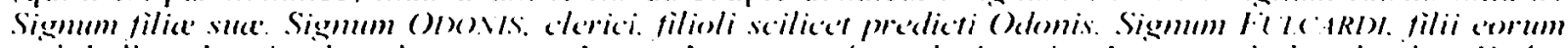
(suit la liste des comoins, dont aucun napparait apparente aux interesses). I a souscription du clere fiudes fait seulement ressortir la parente spirituelle qui le lic au donateur. I a relation de comperage entre la mere

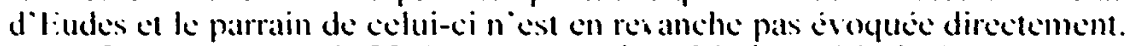

24 Artem n t655. Nous remercions Madame Maric-Jose (iasse-cirandjean de nous asoir fourni ces informations. I. es numeros renvoient a la base de domnes des actes originaux anterieurs a 1121 constitucic par l'Atelier de Recherches sur les lextes Mediciaux (ARTI:M), dont on trouvera un inven-

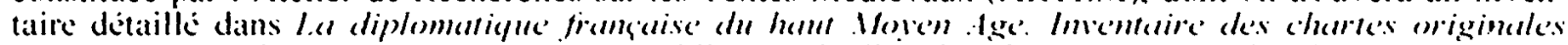

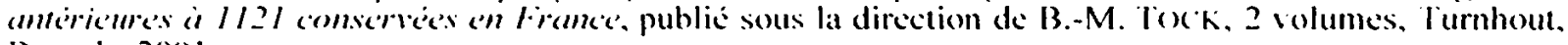
Brepols. 20011 .

25 Artem n 1601 (949), 2567 (984), $2582(991), 4387(104+-1079) .477+\left(X l^{\circ}\right.$ siecle). Sur les

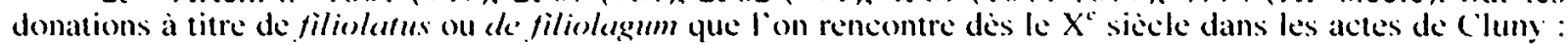
J. l.iveil. "Sprivituale vinculum... ", op. cit. p. 189 et note 46.

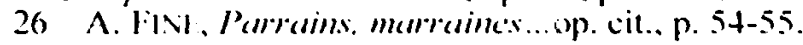


die. la "révolution anthroponymique " des XI"-XII" siecles"7. De ce point de vue, il faut tenir compte non seulement de la binomie attestec de certains individus $\$ \$$. mais aussi du fait que le nom donné au moment du baptême a pu etre éclipsé par une autre dénomination usuelle. Mathilde, épouse d'llenri $f^{\text {er }}$ Beauclere avait ainsi pour nom de baptême lidith, qui la rattachait à la dynastic royale anglo-saxonne dont elle Etait issue par sa mere Marguerite d'Ecosse"2. Plus explicite encore, aux dires d'Orderic Vital, le prince d'Antioche. Bohémond, fut ainsi appelé lorsque son père entendit conter les exploits du géant Bohémond, bien que le nom de baptême de l'enfant ait été Marcio. On ne peut guère juger de la fréquenee de cette praticyue qui semble toutefois aroir été suftisamment arérée pour perturber des cleres chargés de mettre en ordre les archives de leur communauté:

Il est géncialement établi que laattribution au lilleul du nom porté par les parents spirituels ne se répand qu à la lin du Moyen $A$ gé. arec des nuances considérables d'une région à l'autre de l'oceident. (e mode de dénomination semble en revanche moins développé avant le XIII" siecle, bien qu'il soit malaise à évaluer. faute d"une documentation suffisamment explicite sur ex point. Les rares donnés releveses pour le haut Moyen Age suggerent que l’on donnait peut être plus sourent le nom du prêtre yui avait baptise lentant que celui du parrain?. Le cas est encore atteste dans le monde anglo-normand: le lils de Robert Courteheuse, (iuillaume Cliton. refut k

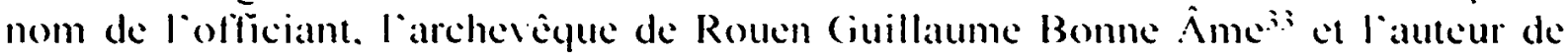

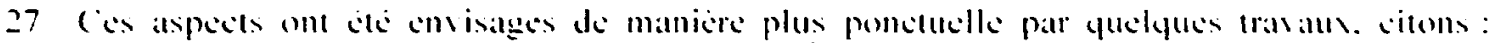

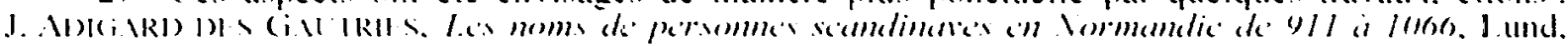

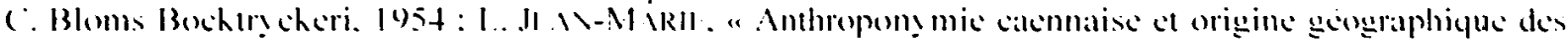

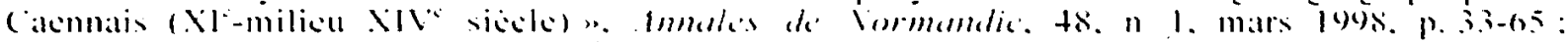

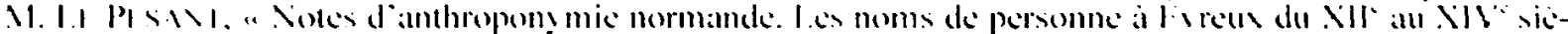

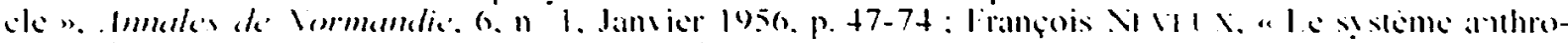

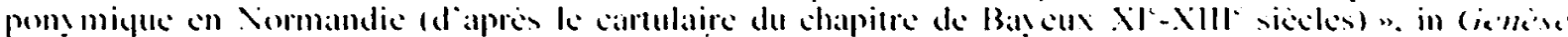

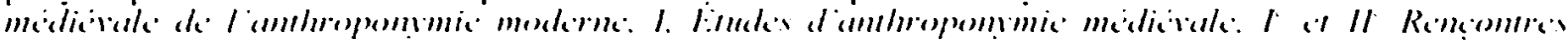

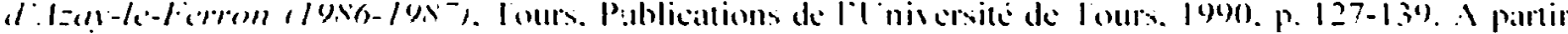

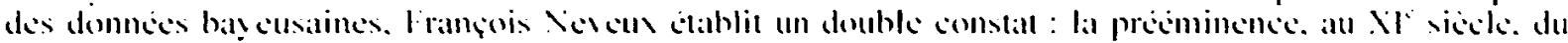

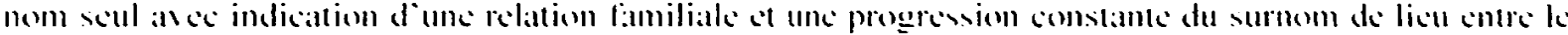

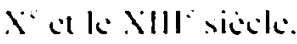

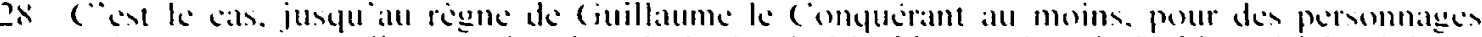

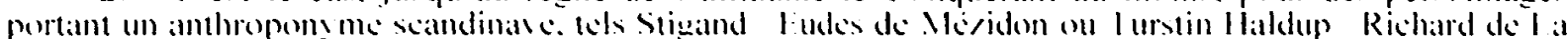

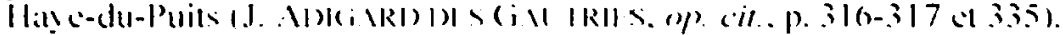

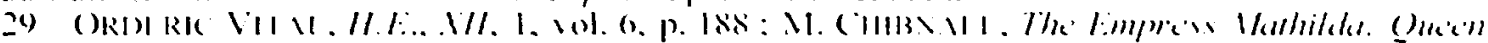

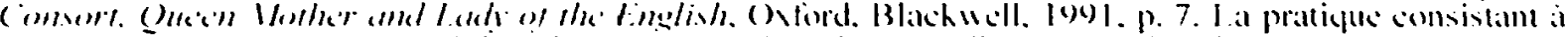

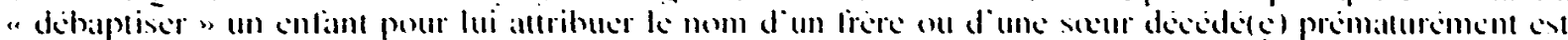

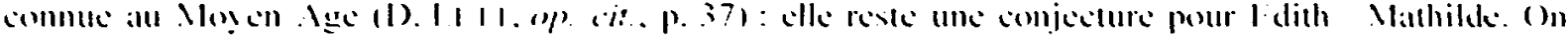

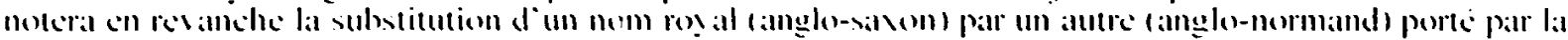

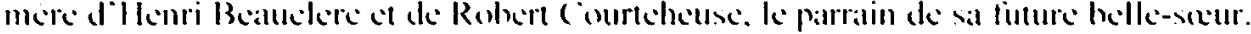

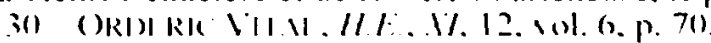

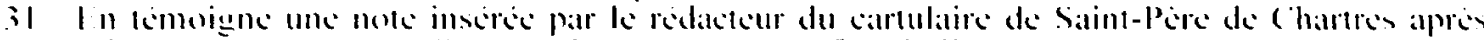

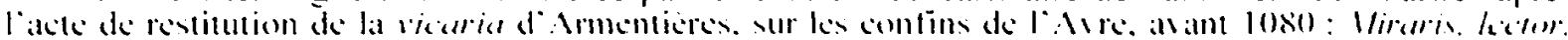

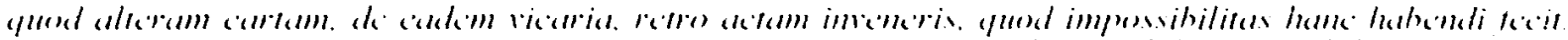

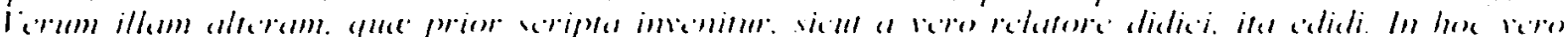

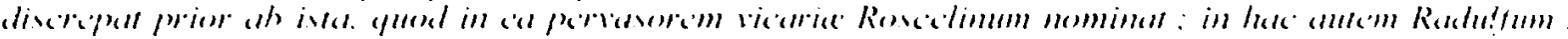

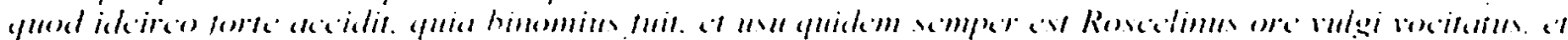

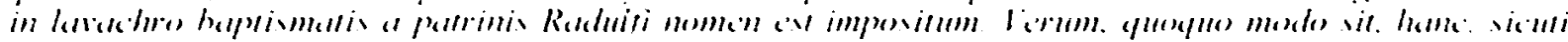

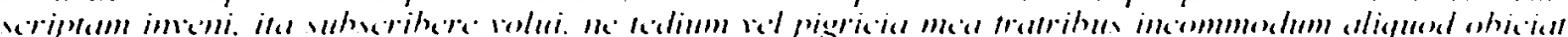

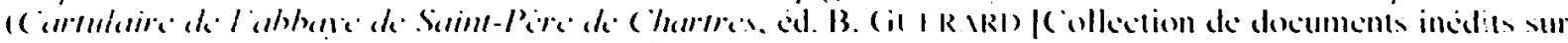
lohistovire de lancel. Paris. Isto. t. 1. p. 1421.

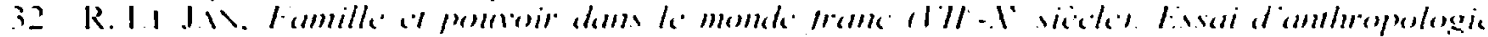
wathe Paris. Publications de la Sorbonme. 1095. p. 189-190.

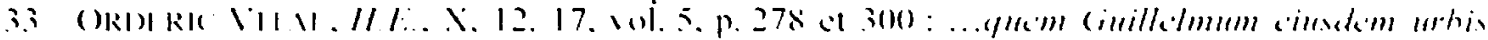

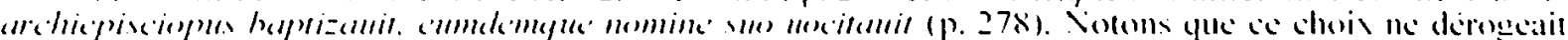

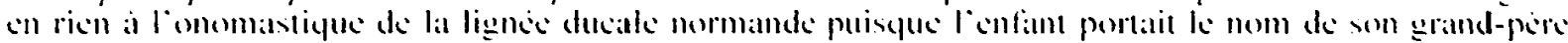
(iuillaumc ḱ (imyucram. 


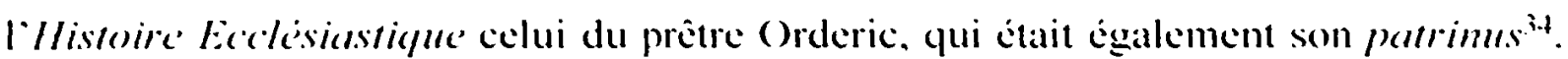
Des exemples dhomonymic parrain/filleul se rencontrent. à commencer pour Rollon/Robert "ou d'autres personnages déja mentionnes tels Thierry, abbe de Saint-livroult, le clere ()don ou Rosedin"s. Ils atlestent une lente diffusion de cette

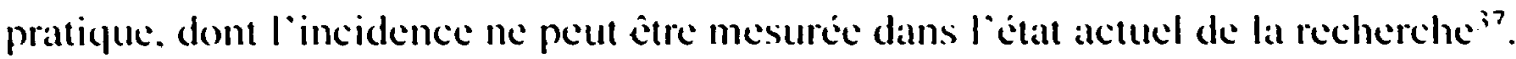

La parenté spirituelle créait - ou était censée établir - des relations deamitié. (etle représentation du parrainage et du comperage est largement éropué dans les sourees normandes. ()rderic Vital souligne. nous l avons vu. kes elfets bénélicpues du lien institue entre Roger du Sap et son compere foulgues : en effet "a partir de ec moment leur commaissance (cognitio) et leur affection (dilectio) s"accrurent peu a peu ". pour le plus grand protit de l'abbaye de Saint-livroult qui put capter la bienveillance du seigneur de (haudry. Dapres Dudon de Saint-Quentin. lorsque Louis IV propose a (iuillaume longue lape de receroir son fils Lothatire des fonts baptismaux. il hait valoir quainsi a ils seront unis par les liens d'une plus garande affection (dilection) et par l"attache d"un amour (amor) plus intense " ". ("est sur ce registre de l"amitic yu aupararant le due (en fait le marcuis de Neustrie). Rohert. softie de devenir le parrain de Rollon car " des lors vous sere\% si cela le com ient, a tout jamais des amis lideles. et nul ne pourra se dresser contre vous et le due accomplira sans relache le serviec yte tu attends de lui et il ferat en sorte yue le roi te soit a tout moment hienteillant " ". Le chel normand repondit favorablement a cette proposition en comparant cette relation spirituelle a lamour yui unit reciprocpuement un pere a son fils. (es tevtes dessent ainsi clairement le cadre dans lequel doient se concevoir. sinon se vive. les liens engendrés par le bapteme : celui de lamitie, voire de l'amour tilial. et d'une étroite affinite d'esprit. A contrario. éest l’image du maurais tilleul qui est d abord retenue par Orderic Vital pour blamer les exactions commises par Richer II de L. Aigle contre Saint-liroulto.

Il ne faut pas sous estimer l’importance de cette representation. l:lle a visiblement ele assey forte pour yu on puisse envisager sous l’angle de la parente spirituelle des relations priviligieses entre deux prinesst. ()n ne setonnera done pas

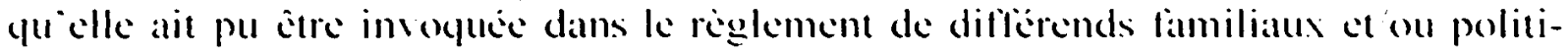
yues. lin 962. Iorsque Robert. pere de (iuillatume de Volpiano. remit la forteresse de Sian (iiulio a ()ton I ${ }^{\text {tr }}$ de (iermanic. la reconciliation entre les deux hommes fut

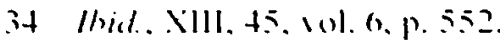

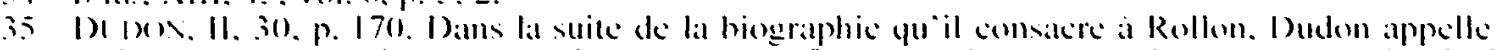

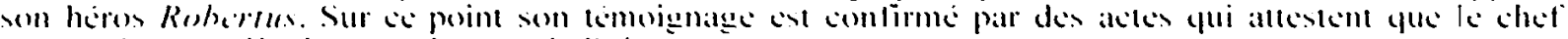

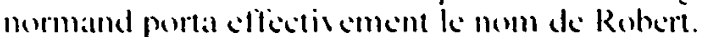

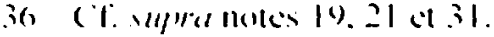

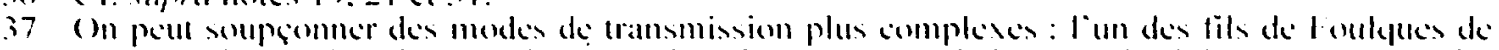

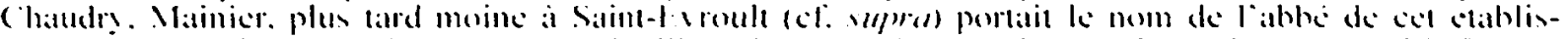

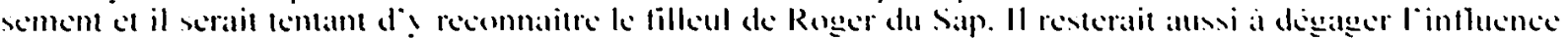

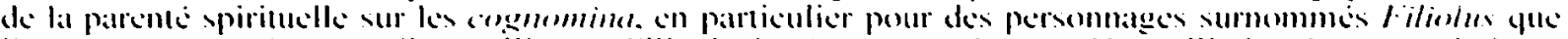

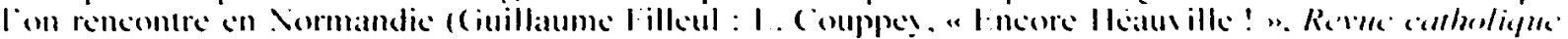

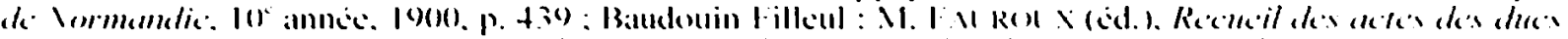

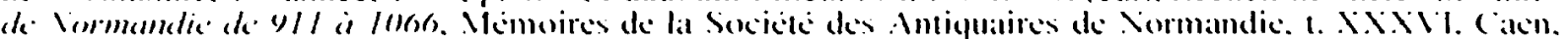

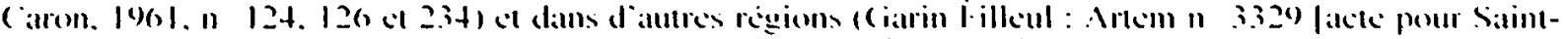

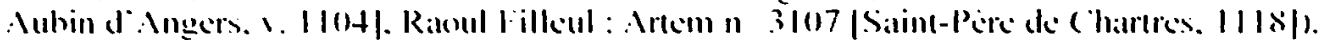

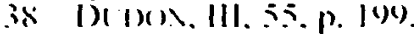

i) 1)1 1) $11.27, \mathrm{p} .167-16 \mathrm{~s}$.

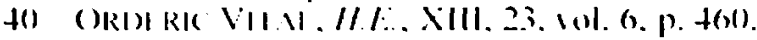

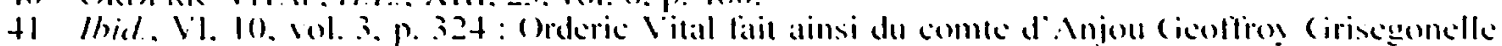

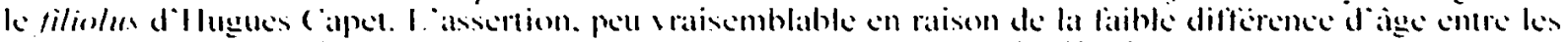

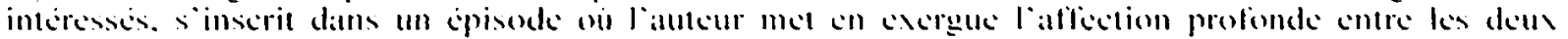
hommes. 
secllec par un accord presoyant de confier le catechuménat du lutur abbe de licamp au couple imperial. Dapres Raoul (ilaber. Fempereur lui donna le nom de (juillatume et lenfant fut reçu des lonts par la reinet2. Ayant imprudemment desarque en Angleterre (1103). Robert courtcheuse fut rapidement contronte a de sombres perspectives, qui lui laissaient le choix entre une captivite imminente et l'abandon d'une rente annuelle de $\mathbf{3 0 0 ( 0 )}$ mares. cue lui avait promise peu auparavant son trère Henri le Beauclerc. Il sauva provisoirement la face grâce à la médiation de sa filleule. Mathilde. épouse du roi d'Angleterre, à laquelle il tit don de la sommet.

La parenté spirituelle offrait ainsi aux processus de pacilication et a des stralegies d"alliance un cadre conceptuel d'atuant plus solide qüil était ancré dans le domaine du sacre. Les travaux d Arnold Angenendt et de Bernhard Jussenth ont montre que les (arolingiens en userent frequemment pour se faire les parrains des rois dont ils avaient obtenu la conversion. parfois apres les avoir laincus. Intersement le parrainage permettait de rétablir a leur protit du moins de maniere symbolique - une situation qui ne leur etait pas farorable. Comme instrument d'harmonisation des rapports de foreses, il fut ansi particulierement sollicite lorsqu il sest age deamener des Normands a la foi chrétienne el détablir arece eux une alliancests. ("est dans ee contexte qu"il faut sans doute replacer loffre de parrainage faite a Rollon par le marquis Robert. Dudon ne cache nullement les enjeux de la belle amitic alors promise par le marquis de Neustric : elle gagnera au futur duc de Vormandice par l’intermédiaire de Robert. la bienseillance du roite. Dans un même

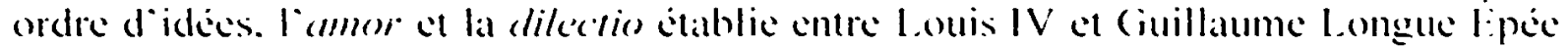
reçoivent une traduction tres concrete lorsque ke prince normand assure son compere de son assistance et de son soutien a lencontre de lous ecus yui oseraient se rebeller contre luit ${ }^{7}$. Parés des altributs de l'amour hilial ou de la confraternite désintéresser. les relations creses par le parramage ou le comperage intestissemt alinsi sans complese le champ des pratiques qui soudent l'amic iria des groupes aristocratic|ues.

Vous arons pu souligner par alleurs la portec politicue du parraninage du marquis Robert : elle marquait non seulement laceptation de Rollon parmi les princes

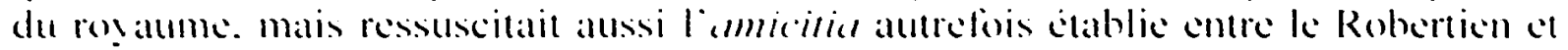

42 like demmi II

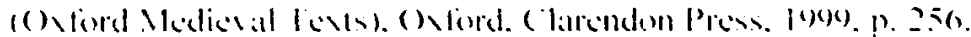

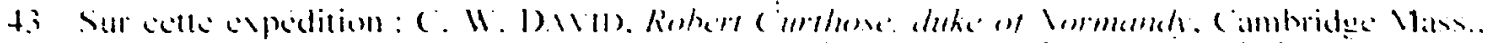

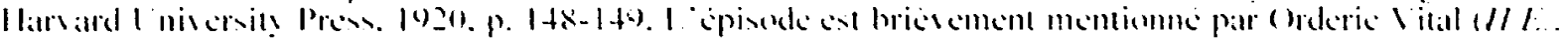

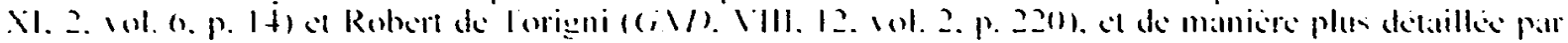

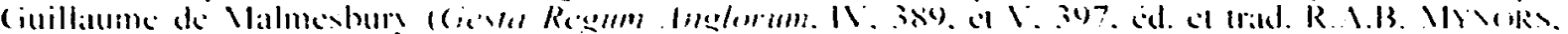

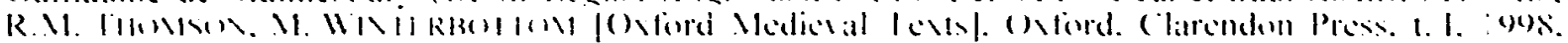
P. 704 o 71 .

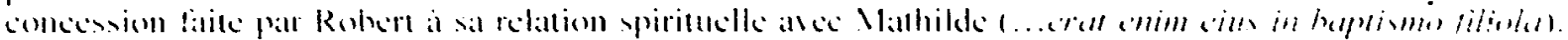

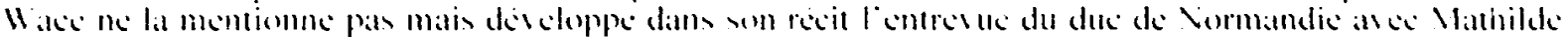

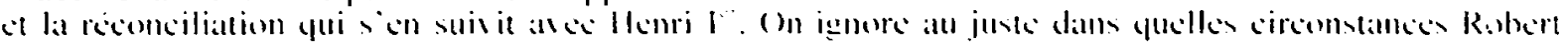

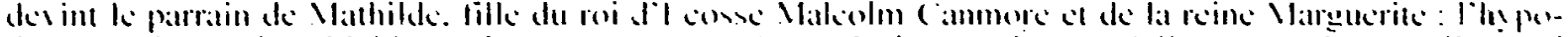

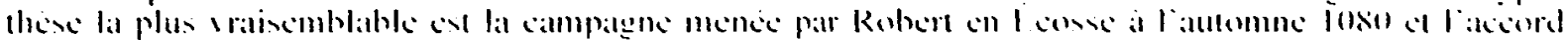

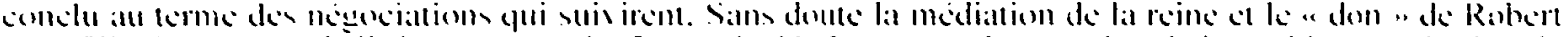

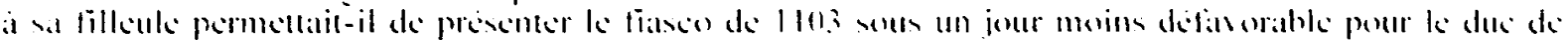

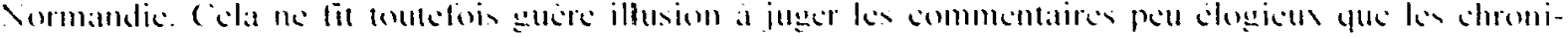

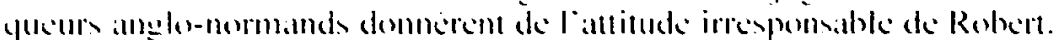

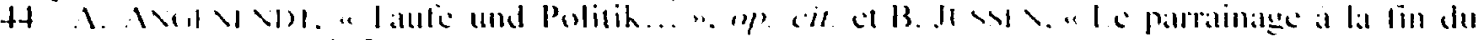

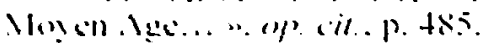

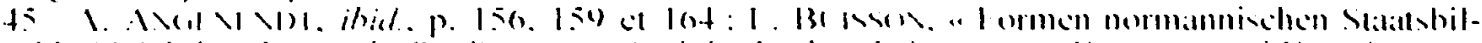

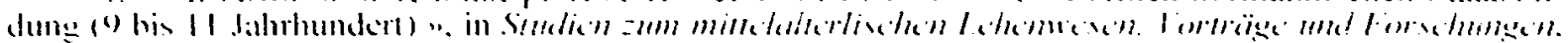

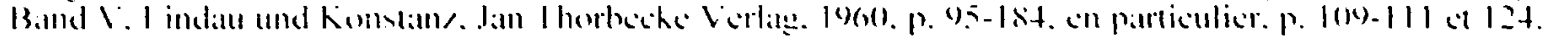

to $131 x(11.27 \cdot 6 \cdot 167-165$.

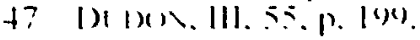


10 marquis Beranger, probable gardien d'une marche de Neustric etablic depuis k

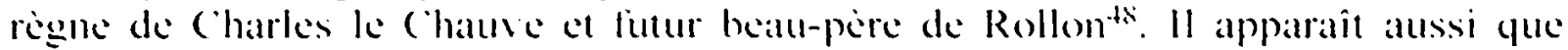
loffre de Robert sinscrit dans un rituel de pacilication, peu apres la délaite des Nomands sous les murs de (hartes et à un moment ou se deroulaient les négociations qui allatent conduire a l'aceord de Saint-( lair-sur-lipte. I.e hat souligne Egalement de maniere remarquable les rapports de forec yui se somt établis en frame du Nord. en particulier pour la région comprise entre Sedne el lovere (e n est pas Charles le simple yui se propose de devenir le parrain du chef normand. mais un prince lemitorial desenu incontoumable dams le reglement de lattaire normande. La recommandation de Rollon ne se double pas deune demonstration symboligue de suprematic qui. par leintermediaire du rite baptismal. aurait lait de charles le "pire" du prince nomand. I. "harmonisation du rapport de force s"opere ici au prolit de Robert".

I.e compinage induisait egalement des relations de solidarite el d’amitic. Cellesci trousatem le mogen de sexprimer a dibientes oceasions. en particulier au moment de la mont deun compire. Ainsi. selon ciuy d Amichs. apres la bataille

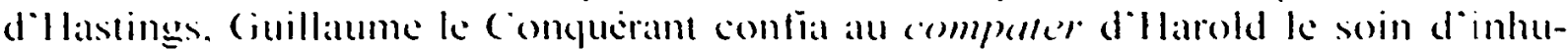
mer k roi delunt". Mais cest datantage l"inkêt d’un comperage prestigieux qui

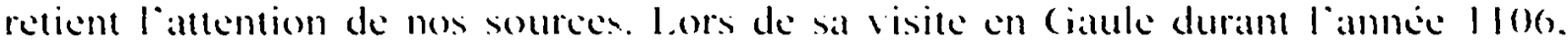
Bohemond d'Antioche vit venir a lui de nombreus nobles lai demandant de conir leur enfant sur les fonts baptismaux". Pensec sur un mode Éalitaire. la relation plaçait. du moins șmbolicuement. les interessés sur un même plan. y compris lors-

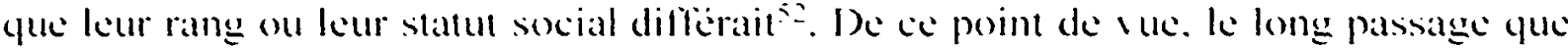
Dudon consace a la demande de comperage faite par louis IV a (juillaume longue

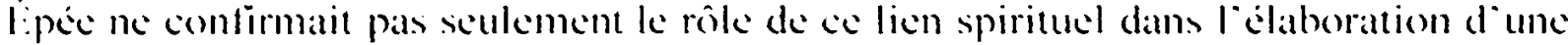
stratsgie d"alliance mais projetait le prince normand sur un pied degalite ar ece le roi.

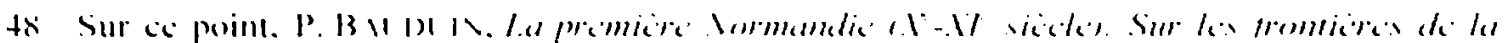

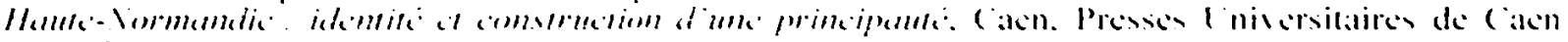
(i) paraitios.

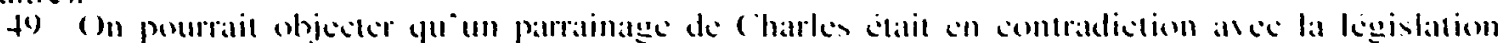

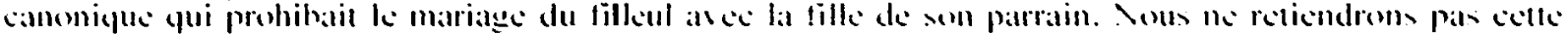

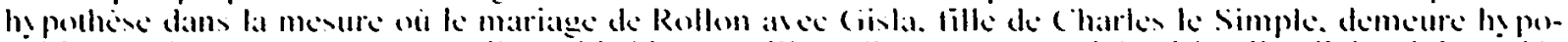

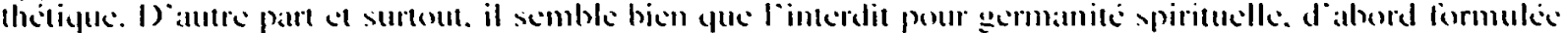

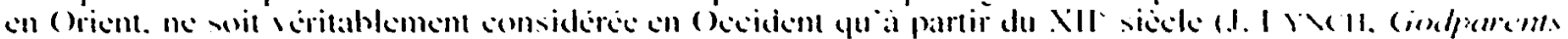

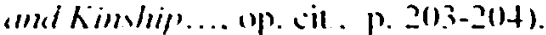

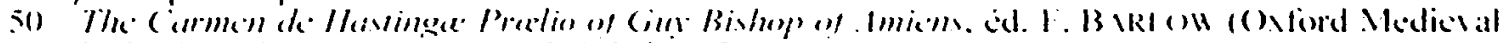

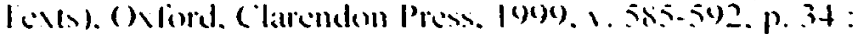

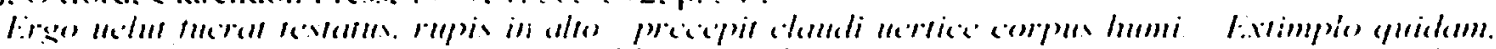

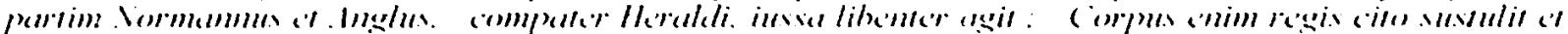

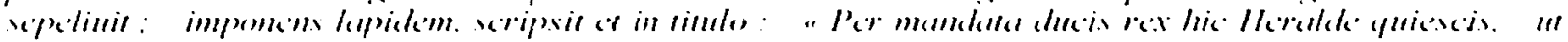

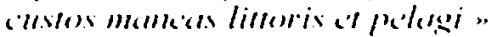

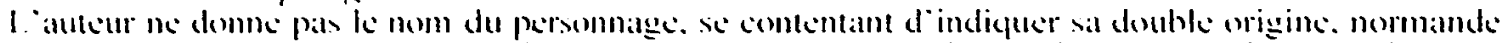

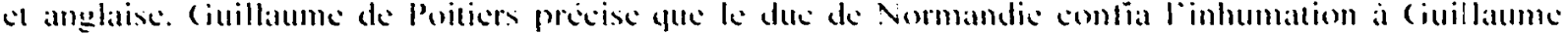

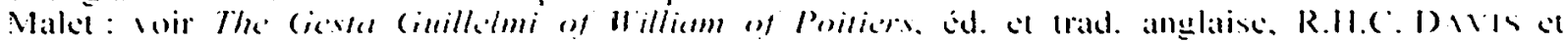

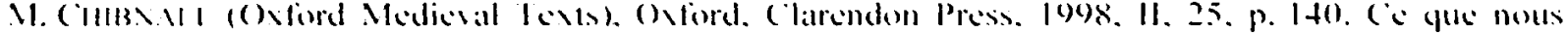

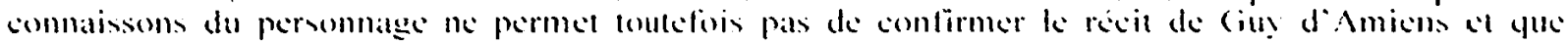

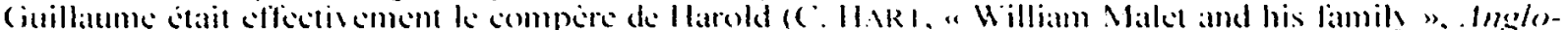

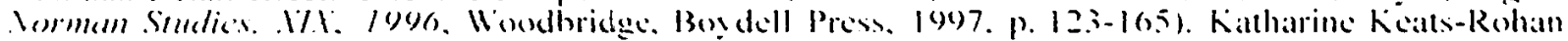

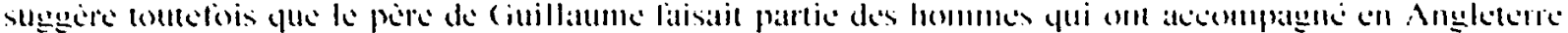

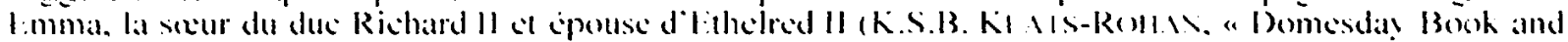

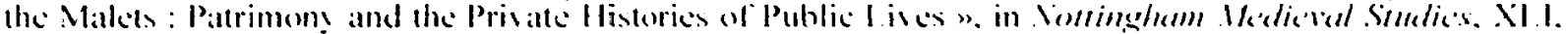
1907. p. 1.3-5.3. en particulice p. 15040.

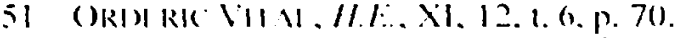

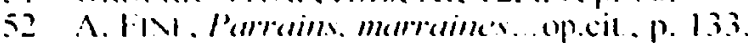


De puissants personnages pouvaient ainsi trouver la l'occasion d'étoffer leur réseau de tideles. Tel fut peut-être le cas de (iilbert de Brionne dans les années 1030. Durant le règne de Robert le Magnifique, cet intluent seigneur issu de la famille ducale tente d'étendre son pouvoir en pays d'Ouche aux dépens des (iéré. établis à Échauffour et à Montreuil-l'Argillés. et de leurs alliés, mais sa première tentative se heurte a une fratrie unie autour de Guillaume (iéré. Gilbert nabandonna pas son projet pour autant et, quelques années plus tard, tenta de prendre le Sap, mais troura la mort dans une embuscade au cours de laquelle furent tués ses comperes Vauquelin de Pont-lichanfré et Foulques (iére ${ }^{5 t}$. Orderic Vital donne deux versions sensiblement différentes de latfaire. Dans une interpolation des Gesta Normannorum Ducum, il relie le meurte à un complot ourdi par Raoul de Gacé et mentionne les assassins, un certain leudes le (iros et Robert (jeré, le frère de foulques, alors que dans l'Histoire Ecclésiastique le chroniqueur évite de porter ectte accusation. qui aurait thetri la mémoire du lignage fondateur de l'abbaye de Saint-livroult. Quoiqu`il en soit. lépisode laisse clairement filtrer les dissensions qui touchent la famille (iéré aux alentours de 1040, et la présence, aux côtés de (iilbert de Brionne. de ses deux compères Vauquelin et foulques mérite attention. Le premier était entré dans le cercle des allies de la famille en epousant íremburge, fille de ciroie l'Ancien. Son beau-frère Foulques (jéré illustre le sort des laissés pour comple de la " mutation familiale" " contraint de virre dans la dépendance du chef de famille (son frère (itullaume). sur une portion restreinte de l'héritage familial (la moitis de Montreuil-l'Argille) et dans l’impossibilite de fonder une ligné légitime (il laissa deux fils issus d une concubine). (e déclassement social créa sans doute un ressentiment propice a des déchirements internes au sein de la famille (iérés. $\dot{A}$ lévidence (iilbert de Brionne réussit a exploiter eette situation, en devenant le eompère de Foulcues et de Vaucuelin. arec le résultat que l'on connatit : une dissociation de la fratric et du réseau d'alliances construit par les (iére en pays d'Ouche. L a affare prit un tour suftisamment critique pour entrainer la réaction brutale qui deboucha sur la mort des trois intiressics.

Les raisons présidant aux choix des parrains de la jeune Alienor Plantagenét en 1161 sont a priori moins evidentes. (On ne peut toutefois exclure quen se donnant pour comperes le tout nouvel évèque d'Atranches, Achard. et l'abbé du Mont-SaintMichel. Robert de Torigni. Henri 11 ait voulu consolider de la sorte son influence dans un secteur stratégique du duché de Normandic, au moment où s'affirmaient ses ambitions sur la Bretagnes.

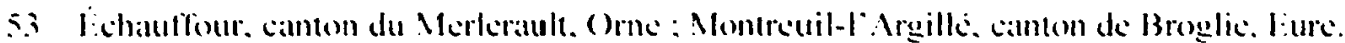

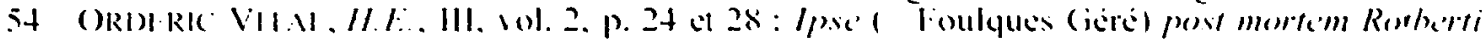

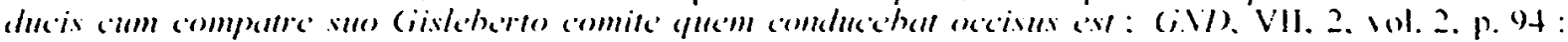

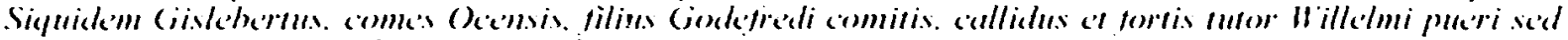

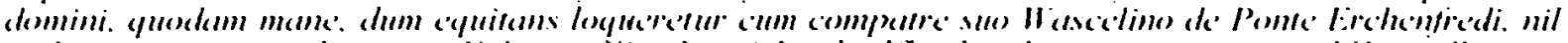

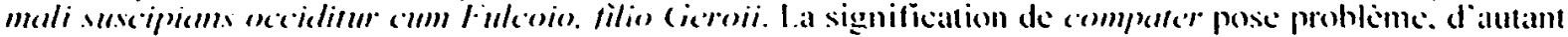

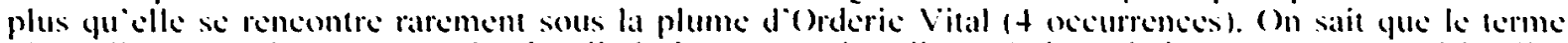

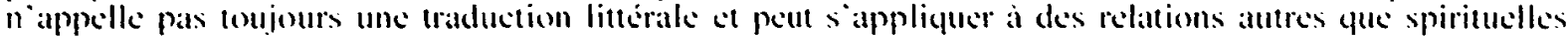

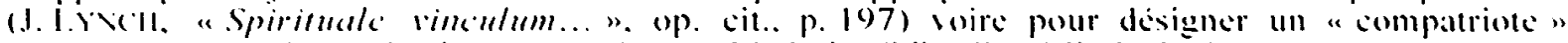
conmmrmam dans les Iraductions proposeses par Marjoric (hibnall el lisabeth Van Ilouts pour cess deux

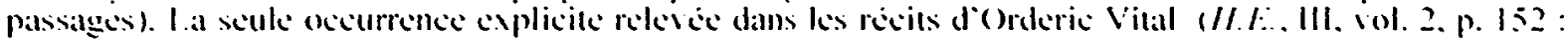

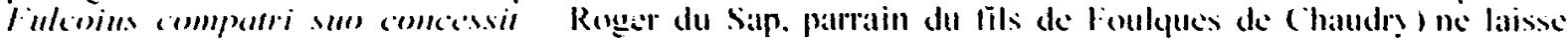
toutefois aucun doute sur la nature du lien de compsiages In dépit deune marge deincertitude. nous conserverons done le sens littsial de somperlor pour interpréter cel spisods.

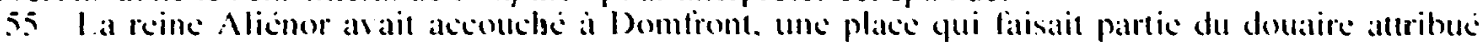

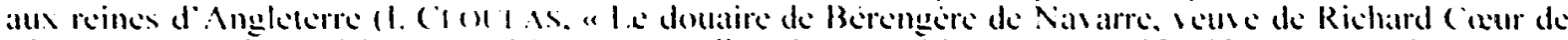

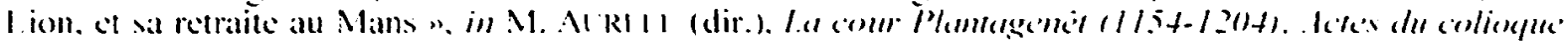


Ces quelques exemples ne doivent pas faire illusion. Sil témoigne bien que la parenté spirituelle occupe une place non negligeable dans les liens sociaux, le dossier réni ne permet pas de mesurer son importance dans les pratiques et les strategies dealliance de la société normande des $X^{\prime \prime}-X I I^{\prime}$ siecles. La discretion des sources constitue en elle-même un motif d'interrogation. Témoigne-t-elle deun desintérêt relatif ou d'une reconnaissance toute marginale de la parenté spirituelle dans l'orbe des rapports sociaux? (ette hypothese serait assurement hasardeuse. surtout paree que l'éclairage apporte sur ee sujet depend en partie d'une documentation dont nous ne disposons pas pour la période, a commencer par les registres paroissiaux. Les sourees judiciaires ou les livres de lamille qui ont fonde létude sur la parente spirituelle pour la lin du Moyen $\hat{\Lambda} g e$. D)autre part il est a remaryuer, si lon sen tient aux seuls chroniqueurs, quelle ne recueille pas le mème intérêt. Dudon. tout en nous reviant l’importance du lien spirituel pour les premiers princes normands. l'inscrit dans une trame qui permet de promousoir ses héros. Orderic Vital se montre plus sensible a la nature affective du lien cré et au comportement moral qui en decoule. Robert de Torigni. si attache par ailleurs à reconstituer les gencialogies et les alliances des principaux lignages du duche. n'ivoque guere les parents spirituels. sauf lorsqu il est directement concerne. $A$ lexception de donness qui sont devenues le fonds commun de l'historiographie normande (bapteme de Rollon. compérage de (iuillaume Longue lipese), ce domaine relese pour lui a ant tout d'une mémoire personnelle.

\author{
Pierre BALDLIN \\ liniversite de ('ac'n Basse-Normundic' \\ ('RAIIM O O I:A
}

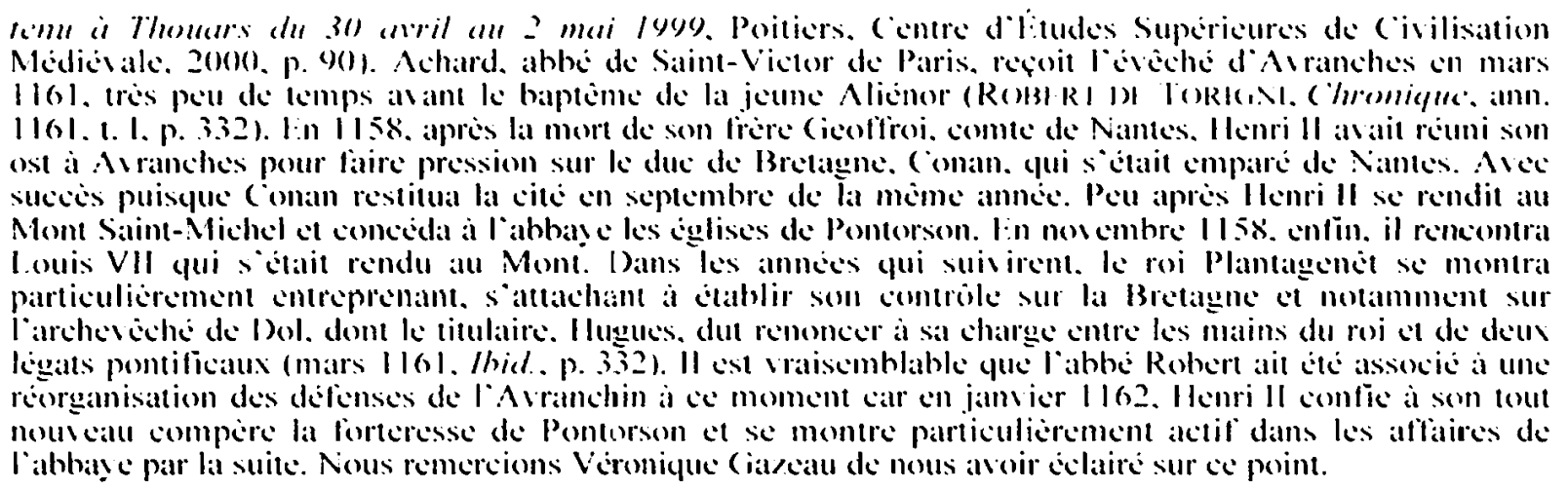

